\title{
Ankle and Foot
}

\author{
Soterios Gyftopoulos and Klaus Woertler
}

\section{Learning Objectives}

- To understand the importance of profound anatomic knowledge for analyzing radiologic examinations of the ankle and foot.

- To get an insight into important ankle and foot pathologies.

\subsection{Tendon Pathology}

\subsubsection{Anatomy and Pathophysiology}

Tendons have one of the highest tensile strengths of all soft tissues but can get injured secondary to both acute and chronic trauma [1-5]. Tendon pathology occurs along a spectrum, beginning with peritendinous inflammatory change. This then can progress to tendinosis, which represents progressive degeneration of the tendon fibers with incomplete healing secondary to chronic stress. Tendinosis sets the stage for tearing, which comes in two variants: partial and complete thickness.

Ankle tendons and any associated pathology can be easily evaluated on imaging, most commonly MR imaging and ultrasound. On MR imaging, a normal tendon appears as an ovoid or circular low signal structure with intact margins. Peritendinous inflammatory change appears as increased T2-weighted signal surrounding portions of the tendon. In instances where a tendon sheath is present, this abnormal signal is consistent with tenosynovitis or sheath inflammation.

S. Gyftopoulos

Departments of Radiology and Orthopedic Surgery, NYU Langone

Health, New York, NY, USA

e-mail: Soterios.Gyftopoulos@nyulangone.org

K. Woertler $(\bowtie)$

Department of Radiology, Technische Universität München,

Munich, Bavaria, Germany

e-mail: klaus.woertler@tum.de
In these cases, you may see associated thickening of the tendon sheath and/or thickened regions of synovium interspersed in the increased T2 signal. Tendinosis will appear as abnormal thickening of the tendon with possible associated regions of intermediate increased intrasubstance signal. With partial thickness tears, you will see a defect or fluid-like increased T2-weighted signal region within a portion of the tendon that does not involve the entire cross-sectional area of the tendon. Patients with complete tears have a defect or abnormal increased T2-weighted signal throughout the entire cross-sectional area of the tendon.

On ultrasound, the normal tendon will have a hyperechoic fibrillar appearance [6]. Tendinosis will appear similar to MR imaging with abnormal thickening of the tendon with possible associated regions of intrasubstance hypoechogenicity. With partial-thickness tears, you will see a defect or defined hypoechoic region within a portion of the tendon that does not involve the entire cross-sectional area of the tendon. Patients with complete tears have a defect or hypoechogenicity throughout the entire cross-sectional area of the tendon.

\subsubsection{Ankle Tendon Compartments}

There are four ankle/foot tendon compartments.

Anteriorly, you will find the anterior tibialis, extensor hallucis, and extensor digitorum tendons (a.k.a. anterior extensor tendons). The anterior tibialis tendon is the largest, strongest, and most commonly injured of the three anterior tendons (Fig. 8.1). Acute injuries can occur in normal anterior tibialis tendons secondary to penetration or laceration injuries from a sharp object such as a hockey boot blade [3]. Acute injuries more commonly occur in the setting of chronic tendinosis, typically in older patients in the 60-70 age range [3]. In certain instances, these patients can present to imaging to rule out neoplasm with a mass-like lesion along the anterior aspect of the ankle, which represents the torn, retracted tendon. 

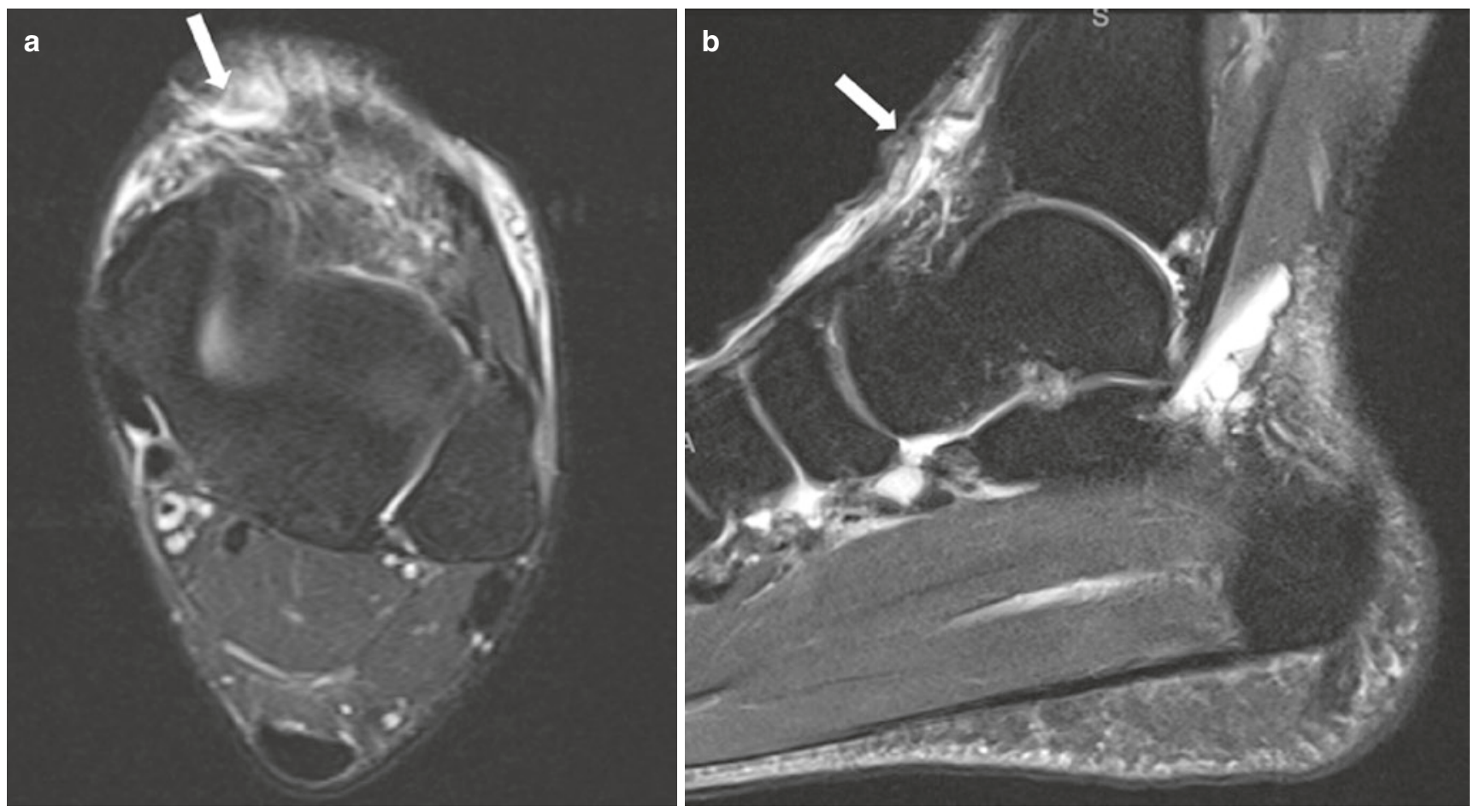

Fig. 8.1 Anterior tibialis tendon tear. Axial (a) and sagittal (b) fat-suppressed T2-weighted MR images of the right ankle demonstrate a fullthickness tear of the anterior tibialis tendon (arrows) secondary to a recent laceration injury

Medially, you will find the posterior tibialis, flexor digitorum, and flexor hallucis tendons (a.k.a. medial flexor tendons). The posterior tibialis tendon is the most commonly injured tendon along the medial ankle, typically in middleaged and elderly women [2]. The spectrum of injuries ranges from tenosynovitis to complete tearing and is commonly associated with flat foot deformity [2]. Partial-thickness tearing of the posterior tibialis tendon can also appear as thinning or atrophy of the tendon [2]. It is important to note that the posterior tibialis tendon can be dysfunctional without evidence of tearing on imaging [2]. The flexor hallucis longus tendon is the second most commonly injured medial tendon, classically seen in dancers.

Posteriorly, you will find the Achilles tendon. The Achilles tendon is most commonly injured $2-6 \mathrm{~cm}$ proximal to its calcaneal insertion, related to the low vascularity in this portion of the tendon (a.k.a. Watershed region) [4]. Injuries are typically posttraumatic in nature with acute tearing superimposed on chronic tendinosis. There are different types of Achilles tendinosis, most commonly hypoxic and mucoid [4]. The insertional portion of the tendon is interposed between the retrocalcaneal bursa (anteriorly) and subcutaneous bursa (posteriorly). These bursae can get inflamed, along with the tendon, in the setting of Haglund's disease (Fig. 8.2) [4].

Laterally, you will find the peroneal tendons: brevis and longus. Peroneal tendon pathology can occur in the acute setting, secondary to ankle sprains, as well as in the chronic setting, in the form of tendinosis from repetitive stress, impingement from displaced fractures of the calcaneus or

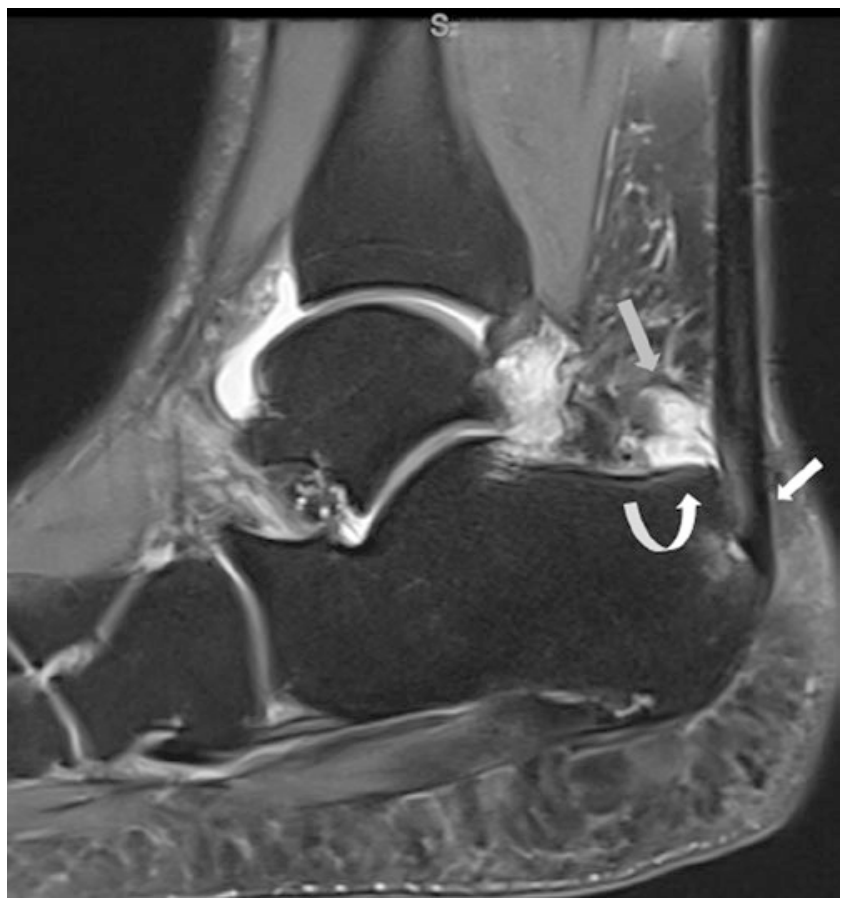

Fig. 8.2 Haglund's syndrome. Sagittal fat-suppressed T2-weighted MR image of the right ankle demonstrates insertional Achilles tendinosis (white arrow) with adjacent retrocalcaneal bursitis (gray arrow) and an enlarged posterosuperior calcaneal margin (curved arrow) in a ballet dancer with chronic posterior ankle pain

distal fibula, or inflammatory arthroplasty [5]. Stenosing tenosynovitis can be seen along the peroneal tendons, secondary to chronic friction along the tendons related to 

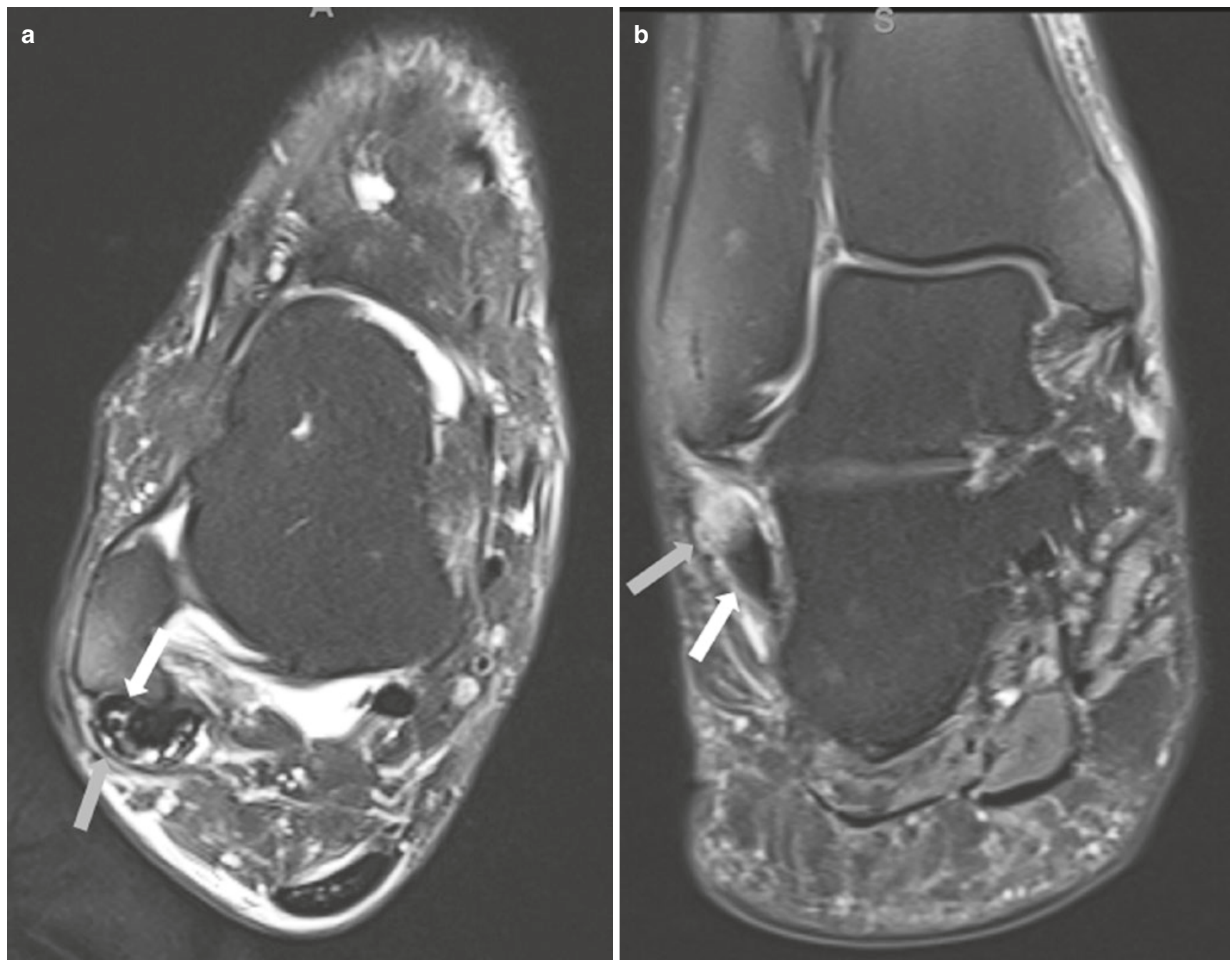

Fig. 8.3 Peroneal stenosing tenosynovitis. Axial (a) and coronal (b) fat-suppressed T2-weighted MR images of the right ankle demonstrate peroneus brevis and longus tendinosis with partial-thickness tearing (white arrows), surrounding tendon sheath thickening and scarring (gray arrows)

tendinosis, thick inferior retinaculum, and/or a narrowed inferior osteofibrous tunnel (Fig. 8.3). Peroneal tendon instability can also be seen on imaging, typically related to superior peroneal retinacular injuries [5]. While displaced tendons can be seen on MR imaging, ultrasound can nicely demonstrate the unstable tendons during imaging.

\section{Key Point}

- Systematic evaluation of the ankle tendons on MR imaging and ultrasound is crucial in both the acute and chronic pain settings. The spectrum of important tendon pathology ranges from tendinosis to partial-thickness tearing to full-thickness tearing. Tenosynovitis is another important imaging finding to report.

\subsection{Bone Injuries}

Bone injuries are commonly seen at the ankle and foot, especially in the athletic population. The most common class of bone injuries diagnosed on imaging are stress fractures, which occur in two varieties [7-9]. There are fatiguetype stress fractures which result from chronic abnormal stress on normal bone. There are also insufficiency-type stress fractures which result from normal stress on abnormal bone. The imaging algorithm for stress fracture typically begins with radiographs which may appear normal or show early signs of fracture including early callus formation, endosteal sclerosis, and/or fracture line [8]. MR imaging is the most sensitive imaging modality for this pathology, with findings including periosteal and endosteal edema, hypointense fracture line, and/or hypointense callus formation [8]. 

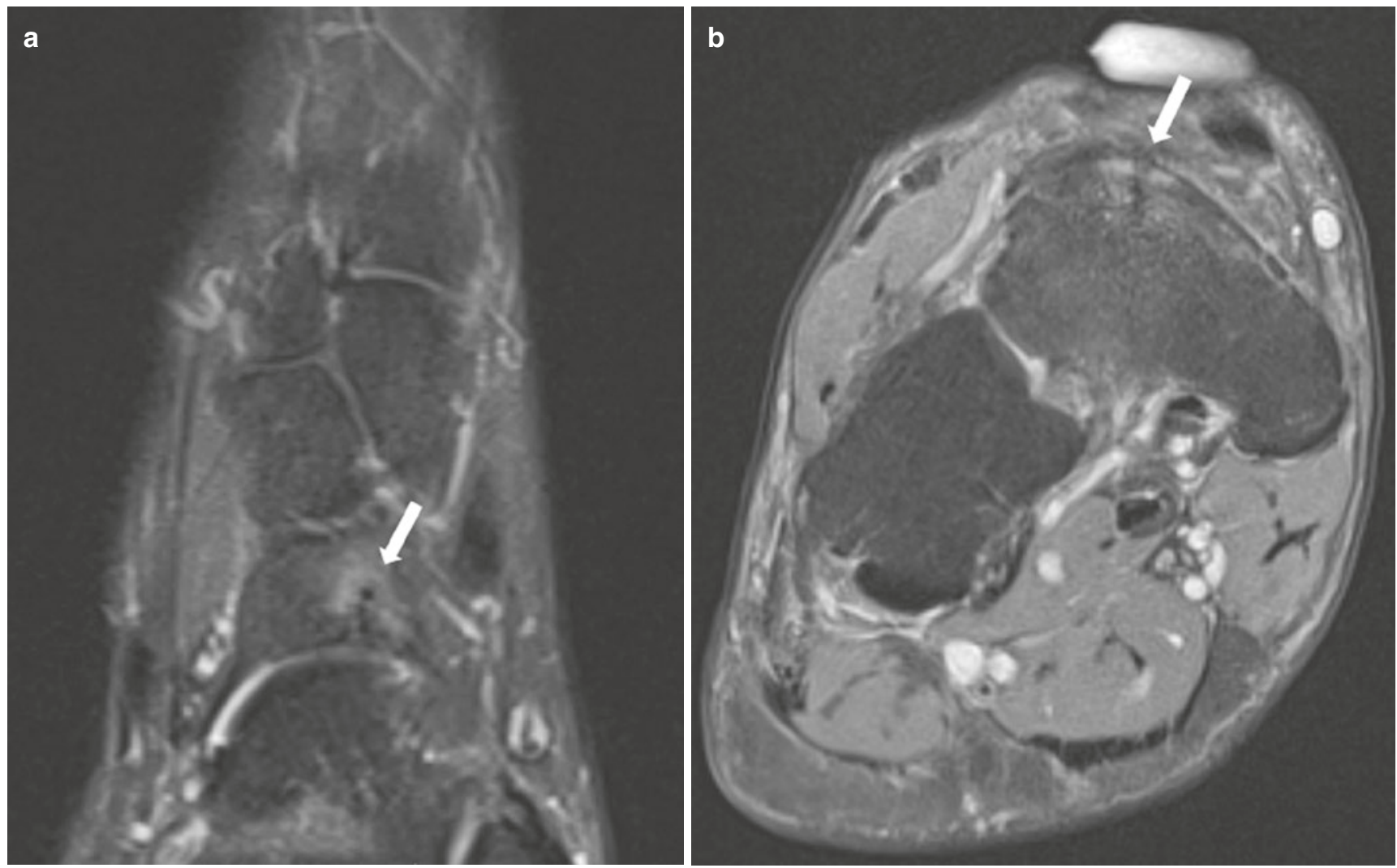

Fig. 8.4 Navicular stress fracture. Axial (a) and coronal (b) fat-suppressed T2-weighted MR images of the left foot demonstrate an incomplete stress fracture with surrounding bone marrow edema along the dorsal aspect of the navicular (arrows)

Common locations for stress fractures at the ankle and foot include metatarsal diaphysis, tarsal navicular, calcaneus, sesamoids, distal tibia, and distal fibula (Figs. 8.4 and 8.5). These injuries are most commonly seen in distance runners but can also be seen in other types of sports including basketball, gymnastics, and ballet [8]. Ultrasound, nuclear medicine, and CT imaging can also be used to diagnose these injuries, although less commonly than radiographs and MR imaging.

\section{Key Point}

- Stress fractures are common and important pathologies to diagnose on radiographs and MR imaging. Familiarity with the most common locations for stress fractures increases your chances for accurate diagnosis.

\subsection{Lisfranc Joint Injuries}

The Lisfranc joint complex compromises three separate compartments: medial, central, and lateral [10]. The medial compartment is made up of the first tarsometatarsal joint, while the central compartment is composed of the second and third tarsometatarsal joints. The lateral compartment is composed of the articulations of the fourth and fifth tarsometatarsal joints. These articulations along with the surrounding capsules and ligaments provide stability to the Lisfranc joint complex.

The most important ligament is the Lisfranc ligament which is found along the medial aspect of the midfoot [10, 11]. The Lisfranc ligament has three components [12]. The strongest and most important component is the interosseous component, which extends from the medial cuneiform to the second metatarsal base $[11,12]$. The second strongest component is the plantar component, which extends from the medial cuneiform to the plantar aspects of the second and third metatarsals. The weakest component is the dorsal component, which is found dorsally and extends from the medial cuneiform to the second metatarsal base.

The imaging algorithm for Lisfranc injury typically begins with radiographs. While gross disruption of the tarsometatarsal joints can be easily seen in the setting of a Lisfranc fracture dislocation injury secondary to high-energy trauma, special attention should be placed on the first and second tarsometatarsal joints in more subtle low-energy trauma cases [13]. One should carefully evaluate the middle cuneiform and second metatarsal base articulation for offset, the 

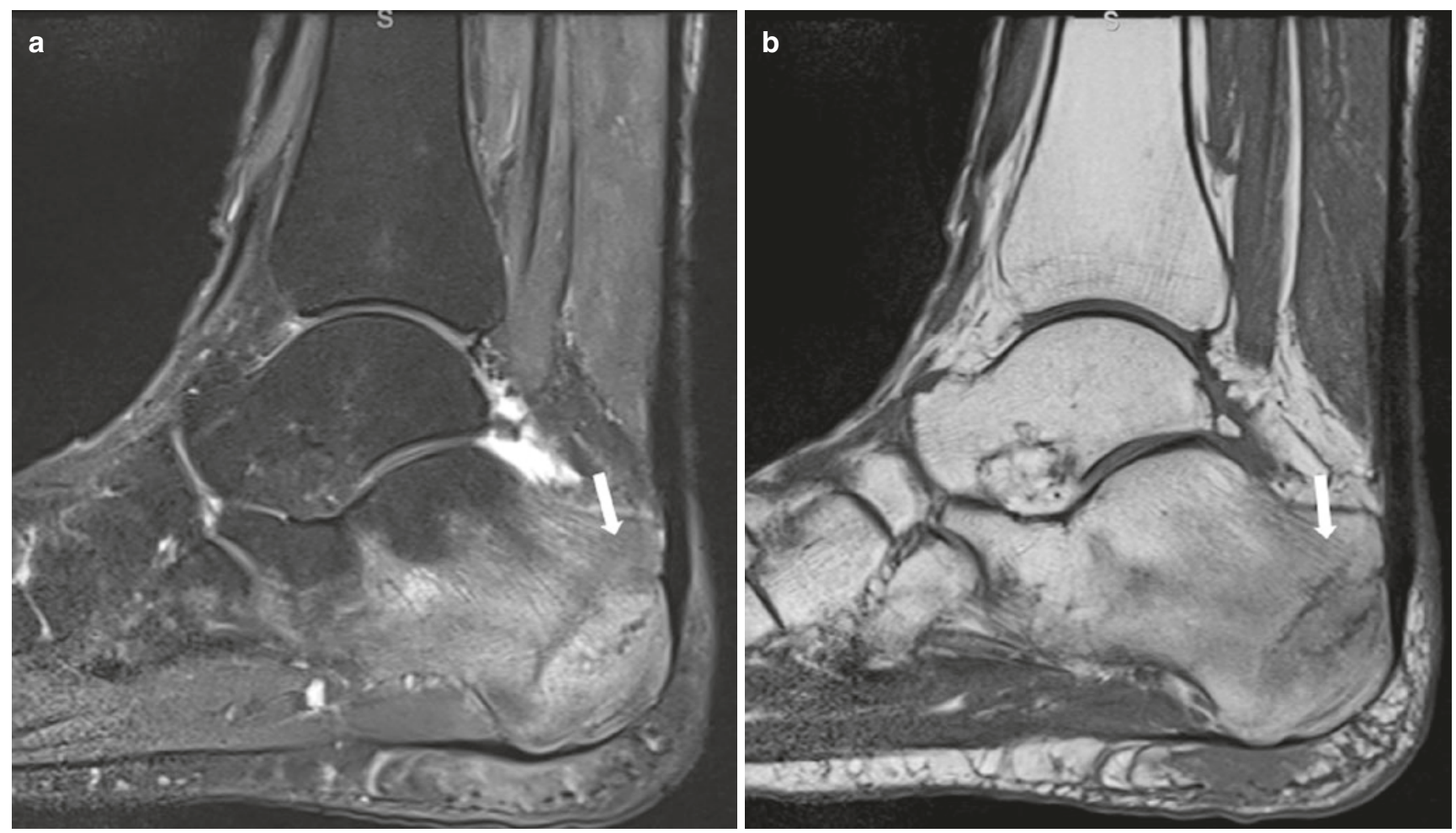

Fig. 8.5 Calcaneal stress fracture. Sagittal fat-suppressed T2- (a) and proton density weighted (b) MR images of the left ankle demonstrate incomplete stress fractures with surrounding bone marrow edema within the posterior third of the calcaneus (arrows)

first and second tarsometatarsal joint interval for abnormal widening $(>2 \mathrm{~mm})$, and avulsion fracture at the second metatarsal base. If possible, weight-bearing radiographs should be obtained as these radiographic findings are more likely to be seen in this imaging setting.

MR imaging is typically the second imaging modality utilized for the evaluation of Lisfranc ligament injury if radiographs are inconclusive and/or more information is needed to make a treatment decision. The main advantage of MR imaging is the ability to visualize each component of the Lisfranc ligament [12]. The normal interosseous component is a thick low signal structure that is best seen on the longaxis and short-axis MR imaging planes extending from the medial cuneiform to the second metatarsal base. This ligament can appear striated, especially on $3 \mathrm{~T}$ imaging, which is a normal variant and should not be confused with pathology [12]. The plantar component is best seen on short-axis imaging as a low signal structure extending from the medial cuneiform to the plantar aspects of the second and third metatarsals. The dorsal component is also best seen on shortaxis imaging as a thin low signal structure extending along the dorsal aspects of the medial cuneiform and second metatarsal base.

MR imaging signs of ligament injury include abnormal increased T2-weighted signal within and/or along the margins of the Lisfranc ligament components, disrupted liga- ment fibers, and avulsion of the ligament from one of its osseous attachments (Fig. 8.6). While all three components should be evaluated, special attention to the interosseous component should be made as the state of this component most directly guides treatment decisions. Bone injuries, including bone contusions and/or fractures, along the margins of the first and second tarsometatarsal joints should bring your attention to the Lisfranc ligament complex in order to assess for possible injury. Focal osseous avulsion fragments can be easily missed on MR imaging; correlation with radiographs or CT would be useful in these settings. CT is also useful, and typically the second imaging modality utilized, in the setting of Lisfranc fracture dislocation injuries.

\section{Key Point}

- High suspicion for Lisfranc ligament injury is crucial in the setting of foot trauma. On radiographs, the reader should pay careful attention to the middle cuneiform-second metatarsal base articulation and the first and second tarsometatarsal joint interval. On MR imaging, the reader should carefully evaluate the interosseous component as this is the most important portion of the Lisfranc ligament complex. 

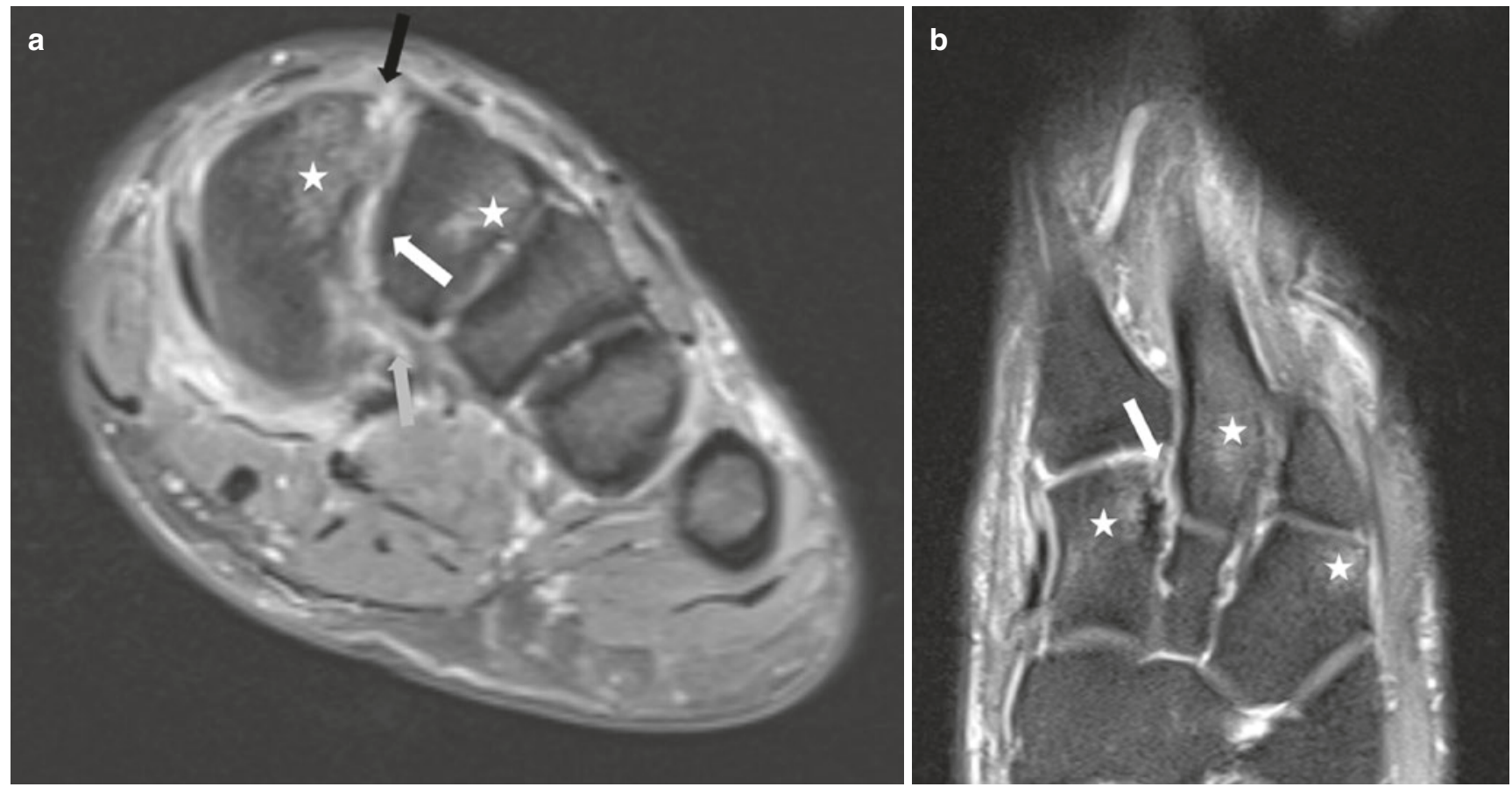

Fig. 8.6 Lisfranc ligament tear. Coronal (a) and axial (b) fatsuppressed T2-weighted MR images of the left midfoot demonstrate tearing of the interosseous (white arrows), plantar (gray arrow), and dorsal (black arrow) components of the Lisfranc ligament and adjacent bone contusions (stars)

paired metatarsosesamoid ligaments. On imaging, each normal ligament appears as a thin structure that extends from the sesamoids to a surrounding structure. Sagittal (ultrasound, long axis) and coronal (ultrasound, short axis) imaging planes are typically the most useful. The most important structures are the sesamoid phalangeal ligaments as they are the most common location for pathology, typically in the setting of hyperextension injury [14]. The musculotendinous part compromises the medial and lateral heads of the flexor hallucis brevis, flexor hallucis longus, adductor hallucis, and abductor hallucis tendons.

Injuries to the first MTP plantar plate complex can occur in the acute (trauma) or chronic (degenerative) setting [14]. Acute injuries, referred to as Turf toe, occur along a spectrum [11, 14]. Mild (grade I) injuries represent sprains and present with abnormal increased T2-weighted signal along the ligament components, most commonly the sesamoid phalangeal, as well as the sesamoids, and/or musculotendinous structures. Grade II injuries represent partial-thickness tearing of the plantar plate complex, most commonly the sesamoid phalangeal, with associated intrasubstance and surrounding abnormal increased T2-weighted signal. Grade III injuries represent complete tearing of the plantar plate complex ligamentous structures and/or fracture or diastasis of the sesamoids. Grade I-III injuries are typically treated conservatively with a positive correlation between injury 

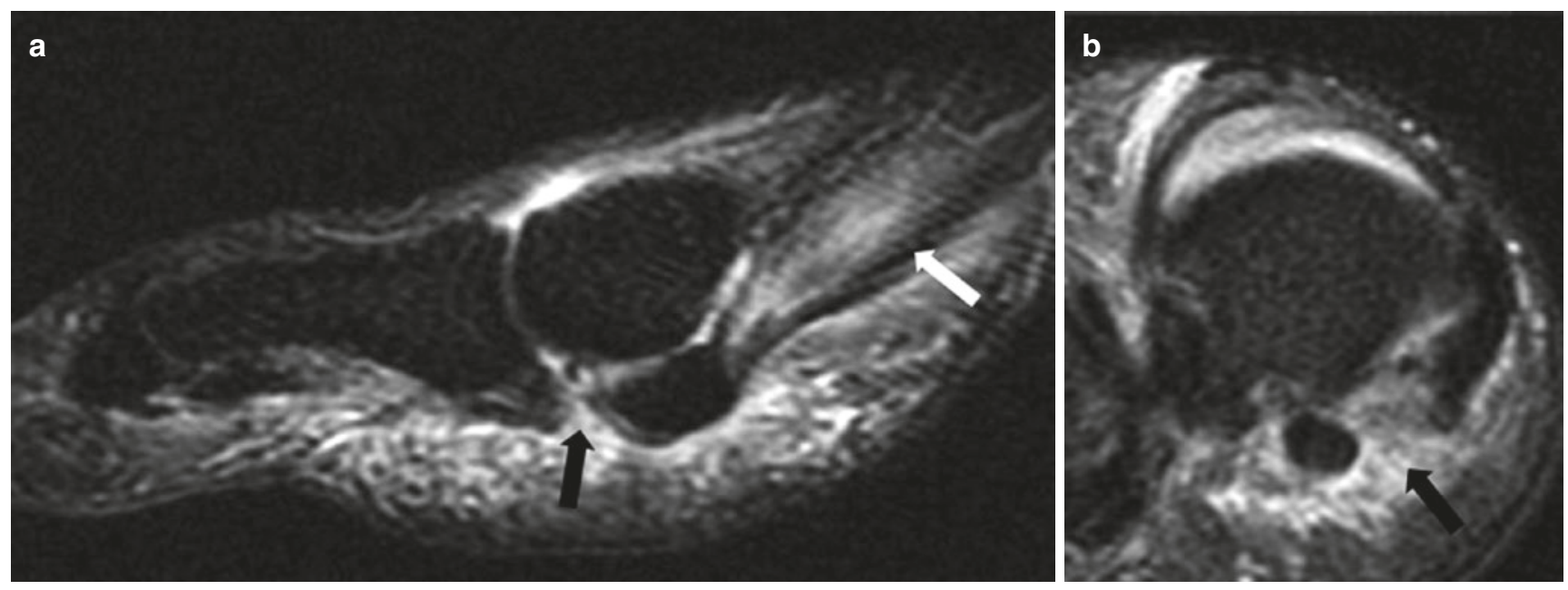

Fig. 8.7 Plantar plate tear (great toe). Sagittal (a) and coronal (b) fat-suppressed T2-weighted MR images of the right forefoot demonstrate tearing of the medial sesamoid phalangeal ligament (black arrows) and strain of the abductor hallucis muscle (white arrow)
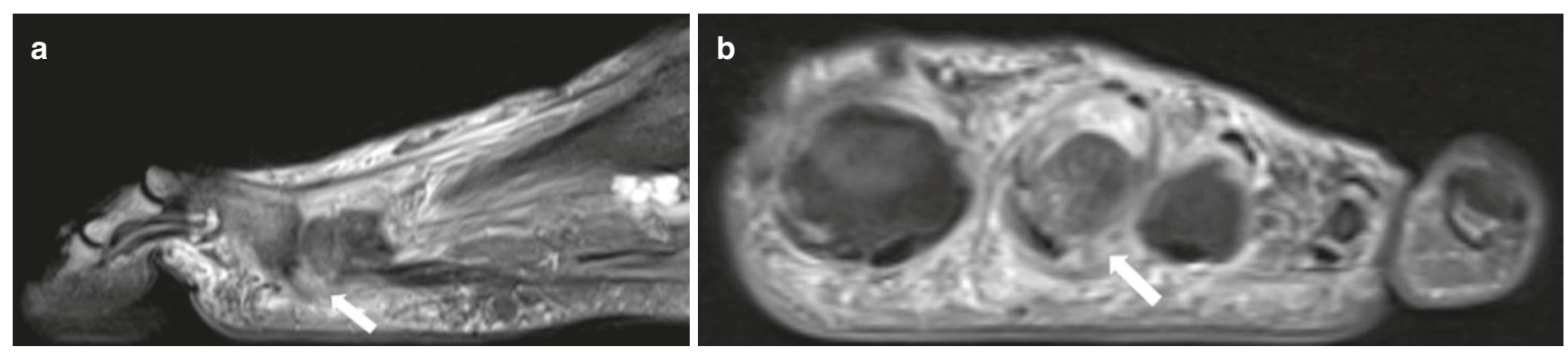

Fig. 8.8 Plantar plate tear (lesser toes). Sagittal (a) and coronal (b) fat suppressed T2-weighted MR images of the left forefoot demonstrate tearing of the second metatarsophalangeal plantar plate along its lateral aspect (white arrows)

grade and recovery time [14]. Marked first MTP joint instability may be an indication for surgery in certain cases [14].

Osteochondral injuries are common and typically involve the sesamoids [14]. Sesamoid pathologies include sesamoiditis (stress reaction), fractures, osteonecrosis, and diastasis of a bipartite sesamoid. Acute injuries along the proximal phalanx and metatarsal head articular surfaces can also occur but are less common. Chronic first MTP plantar plate injuries are typically seen in the setting of osteoarthritis and hallux valgus deformities.

Unlike the first MTP, the plantar plates of the lesser toes consist of a single fibrocartilaginous plate that extends from the metatarsal head (thinnest portion of the plate) to the proximal phalanx base (thickest portion of the plate) at each joint $[11,15]$. Similar to the first MTP joint, the lesser toe plantar plates provide joint stability and articular surface protection. On imaging, the plate appears as a well-defined structure extending from the metatarsal head to the phalanx base. Sagittal (long axis) and coronal (short axis) imaging planes are typically the most useful. The second and third MTP plantar plates are most commonly injured with the spectrum of pathology ranging from degeneration to partialthickness tearing to complete tearing [11]. Plantar plate degeneration appears as thickening of the plate, while tearing typically presents as a defect at the plate insertion (Figs. 8.7 and 8.8). The medial phalanx insertion is the most common initial site for plantar plate pathology. Care should be taken to not confuse the synovitis that can occur adjacent to plantar plate tearing with a Morton neuroma [11].

\section{Key Point}

- Understanding of the plantar plate anatomy is essential for the accurate diagnosis of pathology on imaging. The sesamoid phalangeal ligaments are the most common location for pathology at the first MTP joint. The second and third MTP plantar plates are most commonly injured in the lesser toes with the spectrum of pathology ranging from degeneration to partial-thickness tearing to complete tearing. 


\subsection{Ankle Ligament Injuries}

\subsubsection{Lateral Collateral Ligament Complex}

The lateral collateral ligament complex of the ankle is composed of three main structures: the anterior talofibular ligament (ATFL), the calcaneofibular ligament (CFL), and the posterior talofibular ligament (PTFL). The ATFL, as the weakest of these ligaments, originates from the tip of the distal fibula, takes an almost horizontal intra-articular course, and inserts to the talar neck. The CFL is vertically oriented and extra-articular and extends from the lateral malleolar tip to the trochlear eminence of the calcaneus, lying deep to the peroneal tendons. Both the ATFL and the CFL are seen as linear structures of low signal intensity on MR imaging. The PTFL takes an almost horizontal course from the fibular malleolar fossa to the posterior talus. It has an intra-articular but extra-synovial location and blends with the posterior capsule. On MR imaging, it appears as a strong, often fan-shaped structure with a striated appearance due to fibrofatty composition [16-18].

The lateral ligaments are the most commonly injured structures of the ankle. Approximately $85 \%$ of all ankle sprains result in damage to the lateral ligamentous complex. Inversion injuries most often lead to isolated tears of the ATFL or combined tears of the ATFL and CFL. Isolated tears of the CFL are rare, and injuries of the PTFL almost never occur unless following gross dislocation of the ankle. Typical sites of ATFL and CFL tears are the mid-substance of the ligaments or their insertions to the talus and calcaneus $[16,17]$.

On MR imaging, thickening and increased T1- and T2-weighted signal intensity associated with surrounding edema suggest a partial ligament tear. Complete tears are characterized by discontinuity and retraction of the ligament (Fig. 8.9). Osseous avulsion can be overlooked, in particular if the bony fragment is small, and therefore, correlation with conventional radiography is mandatory. Associated injuries can include osteochondral lesions of the talus; injury to the medial collateral ligament complex, the tibiofibular syndesmosis, the bifurcate ligament, and the ligaments of the tarsal sinus; and peroneal tendon lesions, as well as fractures [16-18].

\subsubsection{Medial Collateral Ligament Complex}

The medial collateral ligament complex has a superficial and a deep layer. The superficial layer crosses two joints and is composed of the tibiocalcaneal ligament (TCL), the tibio-
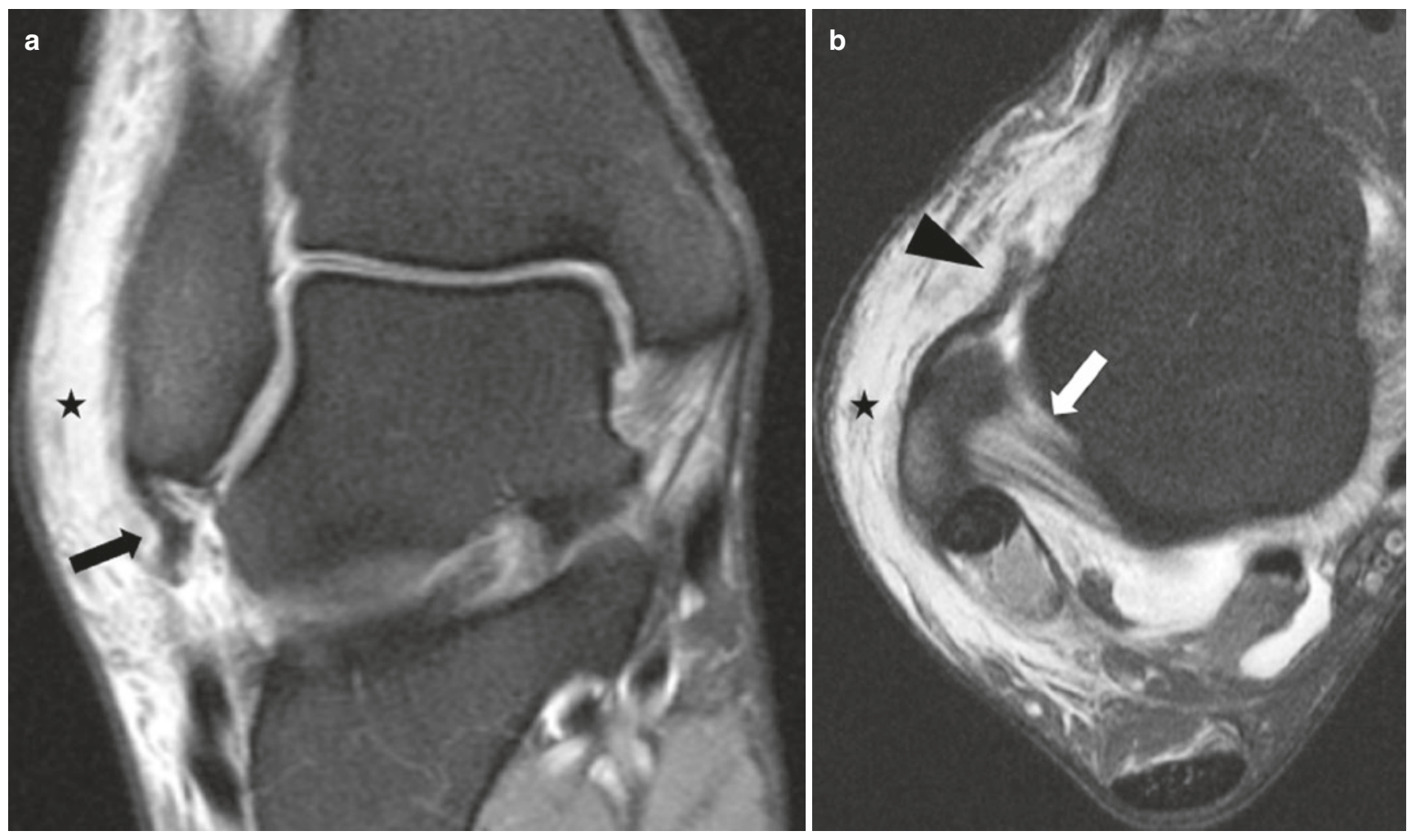

Fig. 8.9 Lateral collateral ligament complex tear. Coronal (a) and axial (b) fat-suppressed intermediate weighted MR images obtained after a right ankle sprain show complete tearing of the calcaneofibular (black arrow) and anterior talofibular (black arrowhead) ligaments with discontinuity and retraction. Note intact posterior talofibular ligament (white arrow) with typical striated appearance. Posttraumatic hematoma is seen in the lateral subcutaneous tissue (stars) 
spring ligament (TSL), and the tibionavicular ligament (TNL). The deep layer crosses only one joint and is formed by the posterior tibiotalar ligament (pTTL) and the anterior tibiotalar ligament (aTTL). Not all of these five components may always be visualized on routine MR images; the pTLL, TCL, and TNL can however be identified in most cases. The pTTL is the thickest of the medial ligaments and differs from the others by usually showing a striated appearance $[19,20]$.

Overall, the medial collateral ligament complex is more resistant to trauma than the lateral one, and therefore, the strength of the medial complex may lead to medial malleolar fracture without rupture of the ligaments. Injury to the medial ligaments is seen in only $5-15 \%$ of all ankle sprains. In more than half of these cases, both superficial and deep components of the medial complex are involved. Most medial collateral ligament injuries occur in combination with lateral ligament tears, malleolar fractures, and syndesmotic tears. Isolated injuries are rare. MR imaging criteria for the diagnosis of partial and complete tears of the different components of the medial complex are identical to those described with lateral ligament injuries. Loss of striation is an additional sign suggesting injury of the pTTL [19-21].

\subsubsection{Tibiofibular Syndesmosis}

The tibiofibular syndesmosis maintains integrity between the distal tibia and fibula and resists axial, rotational, and translational forces. It is formed anteriorly by the anteroinferior tibiofibular ligament (AITFL) and the interosseous ligament (IL) and posteriorly by the posteroinferior tibiofibular ligament (PITFL) and the transverse tibiofibular ligament (TrTFL). The AITFL is often referred to as the anterior syndesmosis, and the PITFL and TrTFL as the posterior syndesmosis. The syndesmotic ligaments have an oblique course, and therefore, some authors recommend oblique axial images for MR imaging. In most cases the tibiofibular syndesmosis can however be sufficiently evaluated by analyzing standard images in all three orthogonal planes. The normal AITFL, PITFL, and TrTFL typically show a fascicular morphology on MR images with T2 contrast [17, 21, 22].

Syndesmotic tears comprise approximately $10 \%$ of all ankle injuries and are often overlooked at initial presentation. They can occur in isolation or in combination with other ligament ruptures. Tearing of the tibiofibular syndesmosis is evident in approximately $50 \%$ of Weber B and the vast majority of Weber $\mathrm{C}$ ankle fractures. A Maisonneuvetype fracture should be ruled out in case of a seemingly isolated syndesmotic injury. In high ankle sprains, the anterior syndesmosis is often ruptured, whereas the posterior syndesmosis remains intact. Posterior syndesmotic injuries are more often bony avulsions from the tibial insertion
(Volkmann fracture) than ligament tears. They commonly occur if all components of the syndesmosis are involved and in combination with ankle fractures [17, 18, 21-23].

Injuries of the tibiofibular syndesmosis can be diagnosed by MR imaging with a high sensitivity and specificity. Partial ligament tears are characterized by thickening, increased signal intensity, and loss of fascicular appearance as well as adjacent soft tissue swelling. Frank discontinuity, a wavy or curved ligament contour, and bony avulsion represent criteria of a complete tear (Fig. 8.10) [22, 23]. Since rupture of the AITFL at its fibular insertion is common, linear high signal intensity on the anterior cortex of the fibula seen on sagittal intermediate or T2-weighted images with fat suppression represents a secondary sign of an anterior syndesmotic tear.

\section{Key Point}

- Evaluation of the ligamentous structures on MR images obtained after ankle sprains is crucial. All components of the lateral and medial collateral complex and the tibiofibular syndesmosis should be routinely checked. Partial tears typically lead to thickening and increased signal intensity of the involved ligaments, whereas complete tears are characterized by discontinuity and retraction. Bony avulsion might be overlooked on MR imaging.

\subsection{Osteochondral Lesions of the Ankle}

Osteochondral lesions (OCL) of the talus (OLT) are most often diagnosed in young adults as sequels of acute or recent trauma. They are estimated to occur in 6-7\% of all inversion injuries, but their true incidence is probably higher. Regarding all sports injuries of the ankle, OLT can be found as associated findings in up to $50 \%$ of the cases. OCL of the tibial plafond are less frequent $[21,24,25]$.

OLT following inversion trauma of the ankle can involve the medial or lateral shoulder of the talar dome. Whereas medial lesions are thought to be caused by compression forces, lateral lesions are more likely initiated by shearing forces. Medial OLT therefore often represent sequels of (osteo)chondral contusions or compression fractures, and lateral OLT are caused by cartilage delamination or (osteo) chondral fractures. All staging systems for OLT are more or less based on the classic publication by Berndt and Harty [26], who classified the appearance of traumatic transchondral fractures: stage 1 describes an area of subchondral compression with intact overlying cartilage, stage 2 a partially detached osteochondral fragment, stage 3 a completely detached osteochondral fragment, and stage 4 a displaced fracture with intra-articular loose body. A stage 5 describing 

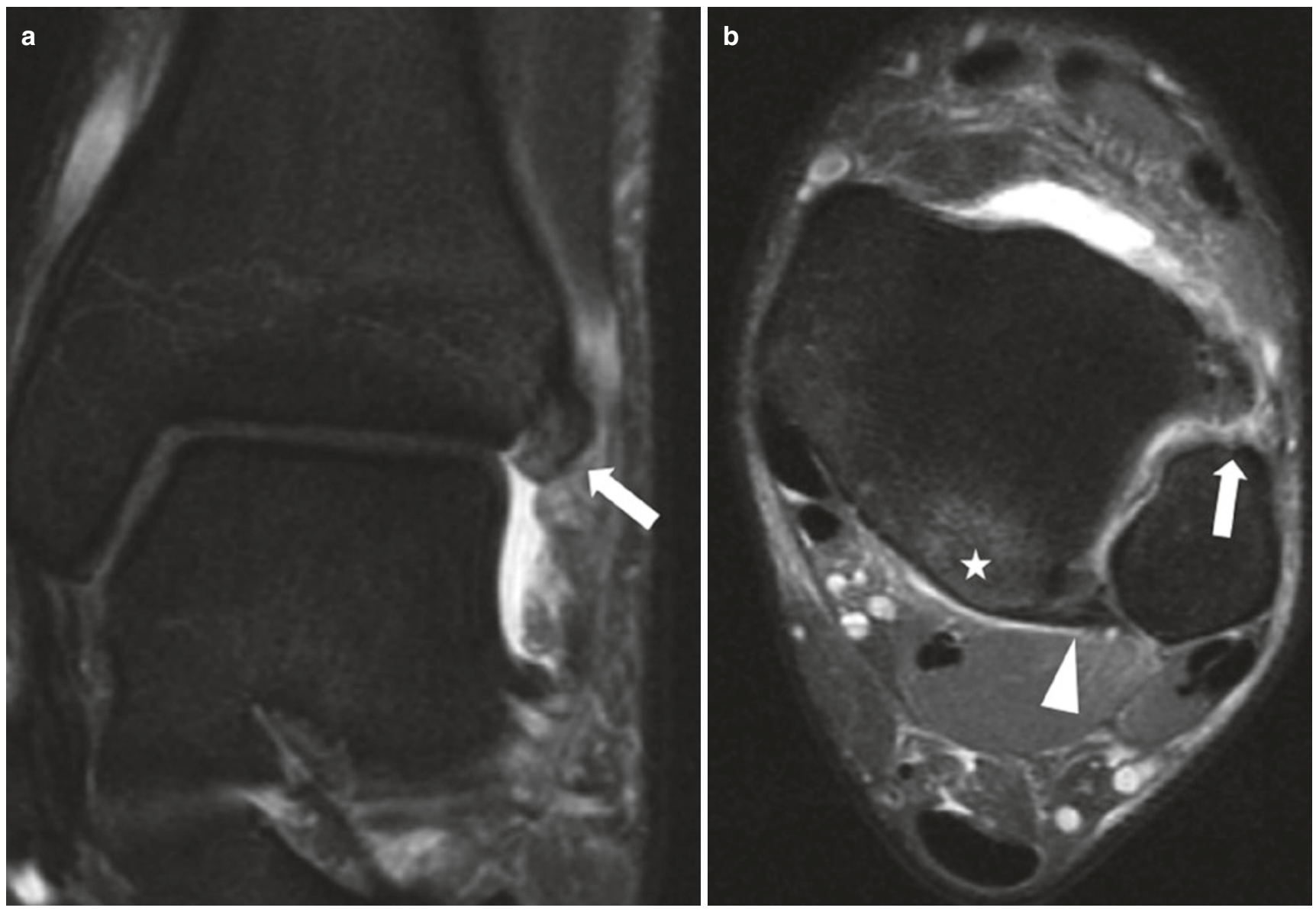

Fig. 8.10 Anterior syndesmotic tear. Coronal (a) and axial (b) fatsuppressed intermediate weighted MR images of the left ankle demonstrate a tear of the anteroinferior tibiofibular ligament at its fibular

formation of a subchondral "cyst" (fibrous defect) was later added by Loomer [21, 24, 26, 27].

In addition to radiographs, conventional MR imaging is usually sufficient to detect and classify OCL of the ankle joint after acute trauma (Fig. 8.11). Chronic lesions are often difficult to characterize on MR imaging due to secondary changes developing in and adjacent to the initially damaged area. In this setting, CT arthrography has proved to be a useful tool to accurately depict cartilage and bone defects of the ankle with an impact on therapeutic decisions [25]. Important criteria for surgical treatment include the integrity of articular cartilage overlying the talar as well as the opposite tibial joint surface, the presence of subchondral "cysts," and the stability and viability of the osteochondral fragment.

\section{Key Point}

- The evaluation of chronic osteochondral lesions of the ankle by MR imaging is challenging. CT arthrography represents a valuable technique for further analysis. insertion (white arrows). The posteroinferior tibiofibular ligament (white arrowhead) has remained intact. Bone marrow edema without evidence of a fracture line is seen in the posterior tibial plafond (star)

\subsection{Ankle Impingement Syndromes}

Impingement syndromes of the ankle are characterized by painful soft tissue encroachment most often due to posttraumatic changes following ankle injuries, usually sprains. The diagnosis is largely clinical and may be supported by imaging findings, including the depiction of morphologic bone and soft tissue changes, localized synovitis, and predisposing anatomic conditions [28, 29]. Anterolateral, anterior, and posterior ankle impingement represent the most important clinical entities. Anteromedial and posteromedial impingement syndromes are less common [28].

\subsubsection{Anterolateral Impingement Syndrome}

Anterolateral impingement is caused by entrapment of abnormal soft tissue in the anterolateral recess (anterolateral gutter) of the ankle, usually subsequent to an inversion sprain with persistent anterolateral pain and swelling. The abnormal soft tissue can represent posttraumatic scar tissue ("meniscoid lesion") or, more rarely, hypertrophy of the 


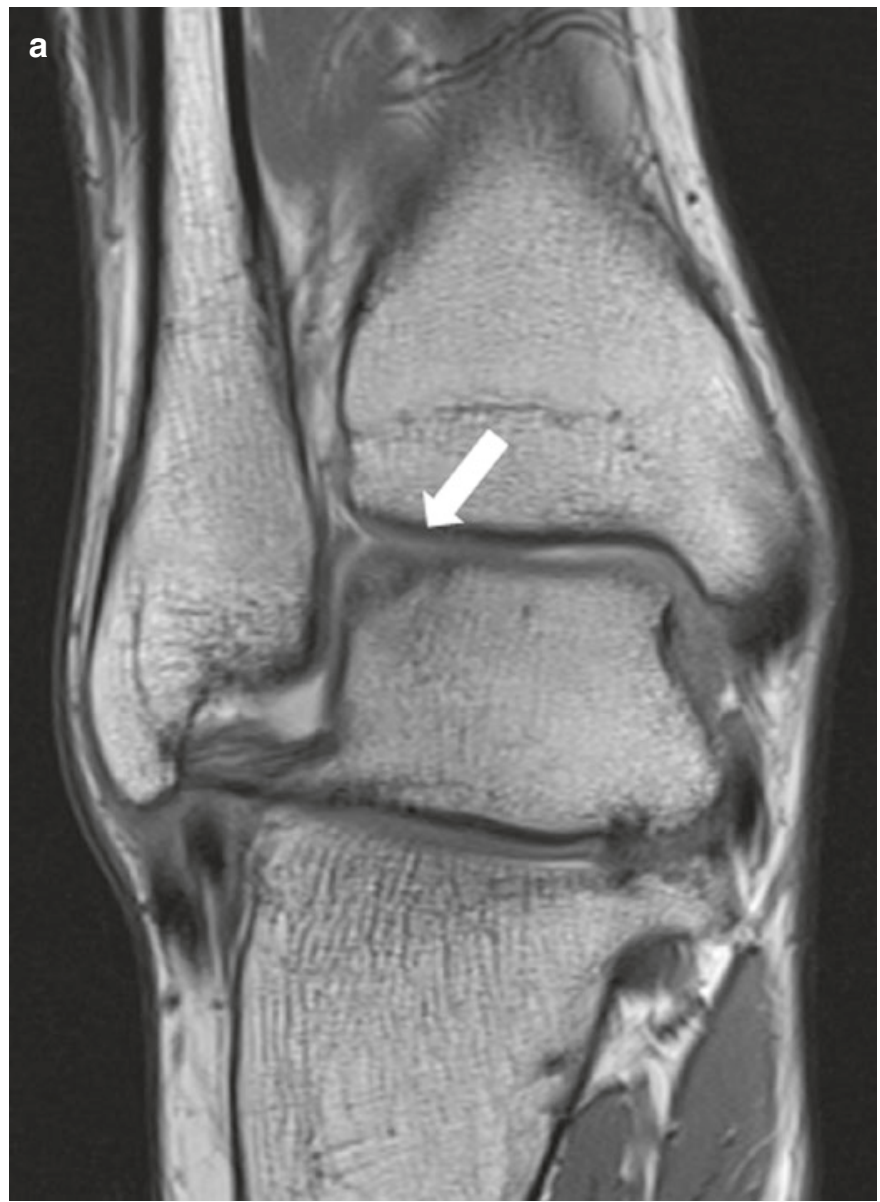

Fig. 8.11 Osteochondral lesion (OCL) of the talus. Coronal T1-weighted MR image with DRIVE pulse (a) and corresponding fatsuppressed intermediate weighted image (b) obtained eight weeks after

Bassett ligament. On MR imaging, scar tissue or synovial hypertrophy can be seen obscuring the anterolateral recess. These findings may be more easily identified on MR arthrography. MR images obtained after intravenous contrast administration can show synovitis exclusively confined to the anterolateral recess [28-31].

\subsubsection{Anterior Impingement Syndrome}

Anterior impingement is relatively common in soccer players, ballet dancers and runners. Tibiotalar spur formation in the anterior joint recess, hypertrophy of capsular tissue, and painful soft tissue entrapment are thought to be induced by repetitive trauma. Conventional radiographs are usually sufficient to identify the underlying bony changes at the distal tibia and the anterior process of the talus. MR imaging can demonstrate bone marrow edema, cartilage loss, capsular thickening, and synovitis in the anterior recess $[28,30]$.

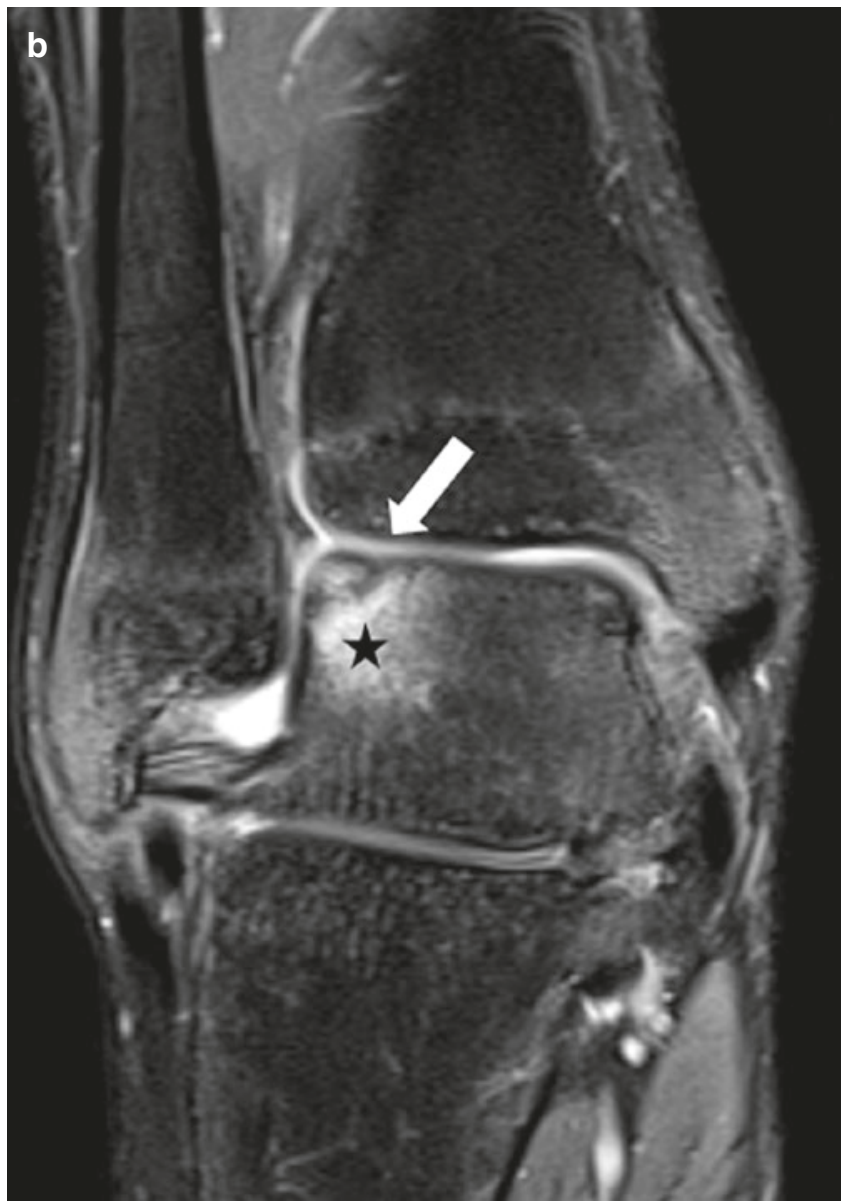

inversion injury of the right ankle show non-displaced osteochondral fracture at the lateral shoulder of the talus (arrows) with adjacent bone marrow edema (star)

\subsubsection{Posterior Impingement Syndrome}

Posterior ankle impingement is typically seen in athletes who execute repetitive plantar hyperflexion, such as ballet dancers and soccer players. The underlying mechanism is compression of bone and soft tissue structures between the tibia and the calcaneus during forced plantar flexion of the foot. Predisposing conditions include the presence of an os trigonum, an elongated Stieda process, and a fractured lateral tubercle of the talus. A more downsloping articular surface of the tibia, a prominent posterior process of the calcaneus, and a posterior intermalleolar ligament may also contribute. Conventional radiography allows for identification of the abovementioned variants and changes of osseous anatomy. MR images can show bone marrow edema in all involved osseous structures, fluid within a non-united fracture, thickening of the posterior capsule, synovitis in the posterior joint recess, as well as tenosynovitis of the flexor hallucis longus and soft tissue edema (Fig. 8.12). An abnormal intermalleolar ligament may also be visualized [28, 30]. 


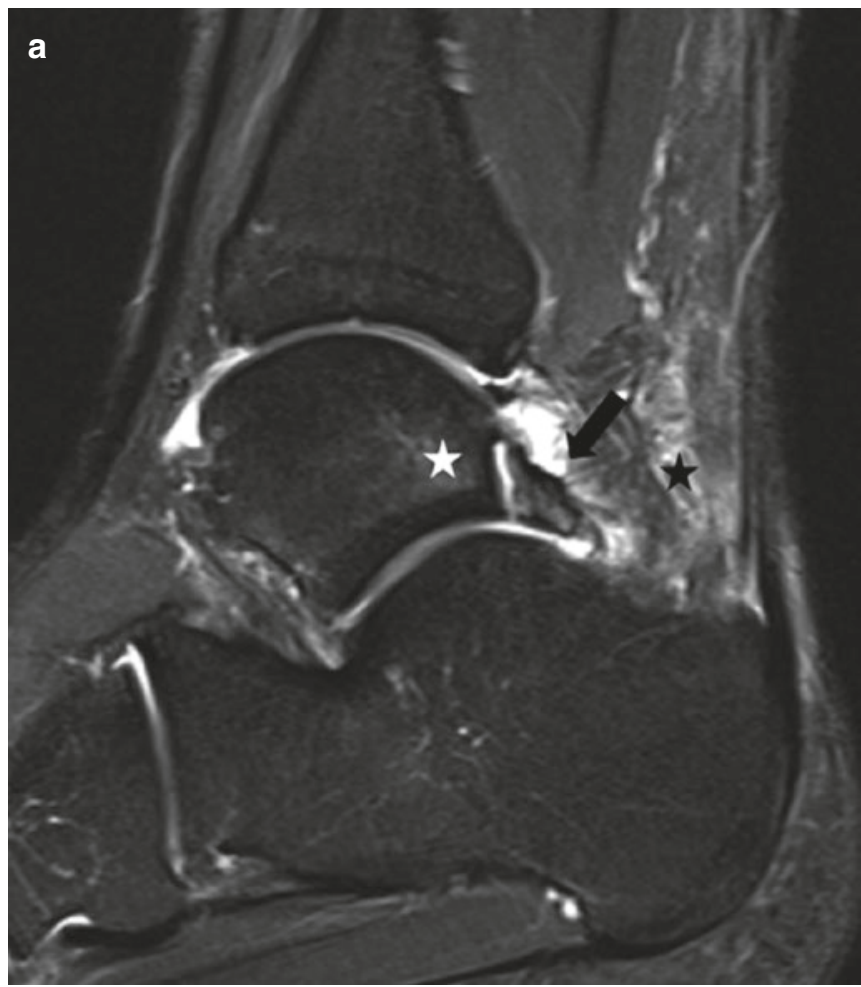

Fig. 8.12 Posterior ankle impingement (os trigonum syndrome). Sagittal fat-suppressed intermediate weighted MR image of the ankle (a) demonstrates os trigonum with bone marrow edema separated from the talus by interposed fluid (black arrow), bone marrow edema in the posterior talus (white star), and retroachillear soft tissue edema (black

\section{Key Point}

- Ankle impingement is a clinical and not a radiological diagnosis.

\subsection{Nerve Entrapment}

\subsubsection{Tarsal Tunnel Syndrome}

Tarsal tunnel syndrome is caused by compression of the posterior tibial nerve and its branches in the tarsal tunnel, a fibroosseous canal extending from the posteromedial ankle to the plantar aspect of the foot. Possible clinical symptoms include pain and paresthesia at the medial heel and/or the medial and plantar aspect of the foot and toes, positive Tinel's sign, and weakness of the plantar muscles. Entrapment of the nerve can occur due to trauma or repetitive stress (athletes), foot deformity, ankle instability, spur formation, and space-occupying masses (ganglia, nerve sheath tumors, accessory muscles). MR imaging may show morphologic changes of the posterior

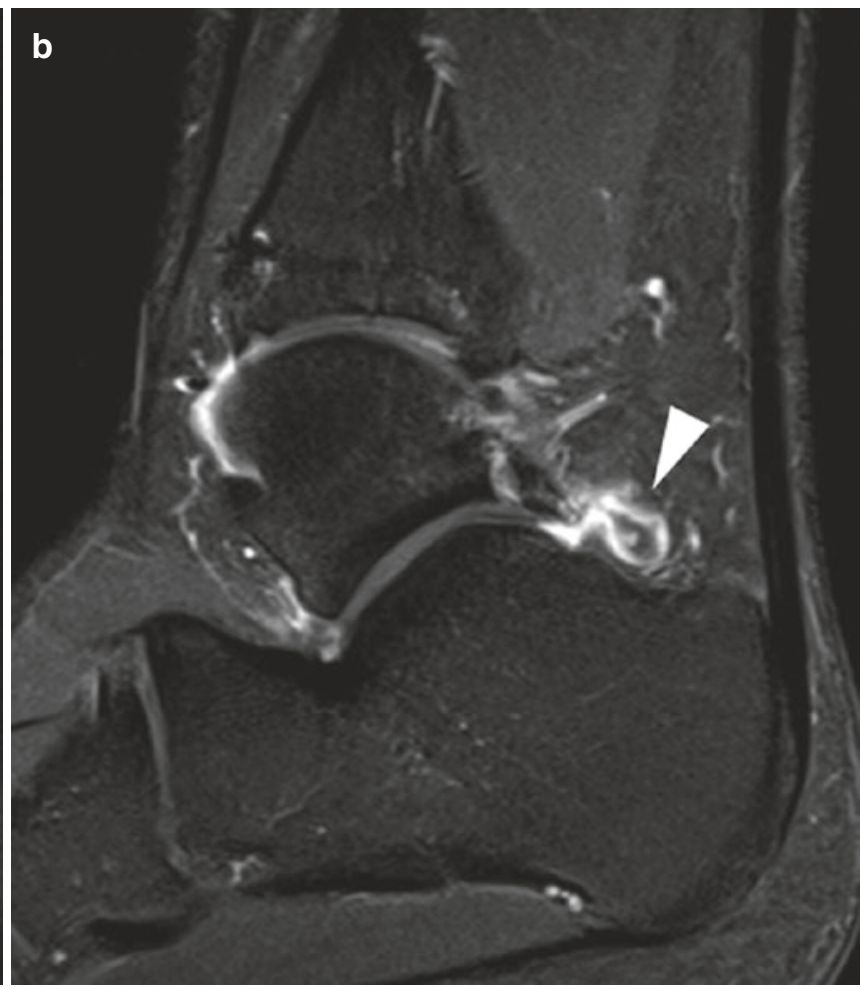

star) in a soccer player with clinical symptoms of posterior impingement. Corresponding fat-suppressed T1-weighted image after contrast administration (b) shows localized synovitis in the posterior joint recess (white arrowhead)

tibial nerve, denervation edema of the plantar muscles, a soft tissue mass (Fig. 8.13), (teno)synovitis, or diffuse contrast enhancement of the tarsal tunnel [32-34].

\subsubsection{Baxter Neuropathy}

The inferior calcaneal nerve (Baxter nerve) is a mixed sensory and motor nerve, which arises from the lateral plantar nerve within the tarsal tunnel. It takes a vertical course between the abductor hallucis and quadratus plantae muscles and then makes a horizontal turn, coursing laterally beneath the calcaneus to innervate the abductor digiti minimi muscle. Baxter neuropathy due to stretching of the inferior calcaneal nerve is a common problem in runners, ballet dancers, and gymnasts, who experience heel pain and burning sensations radiating along the lateral foot. Other causes include a hypermobile pronated foot, hypertrophy of the abductor hallucis muscle, inferior calcaneal spurs, and plantar fasciitis. A classic sign of affection of the Baxter nerve on MR imaging is denervation edema or fatty infiltration of the abductor digiti minimi muscle [32-34]. 


\subsubsection{Jogger's Foot}

Entrapment of the medial plantar nerve between the abductor hallucis muscle and the plantar crossover of the flexor digitorum longus and flexor hallucis longus tendons ("knot of Henry") is classically described in runners, typically with hyperpronation and heel valgus. Clinical symptoms are radiating medial foot pain, tenderness, and dysesthesia along the

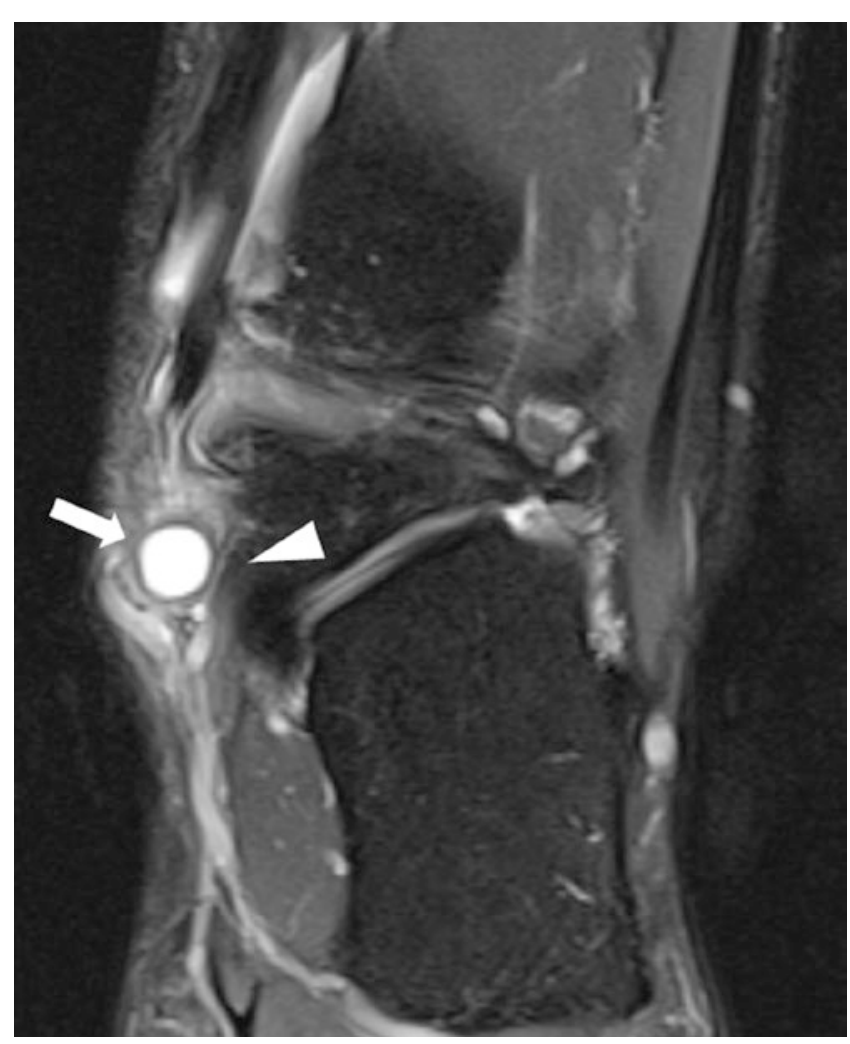

Fig. 8.13 Tarsal tunnel syndrome caused by a ganglion. Coronal fatsuppressed intermediate weighted MR image of the left ankle shows cystic mass (arrow) with surrounding soft tissue edema entrapping the tibial nerve (arrowhead) in the posterior tarsal tunnel. The patient experienced typical clinical symptoms of tarsal tunnel syndrome

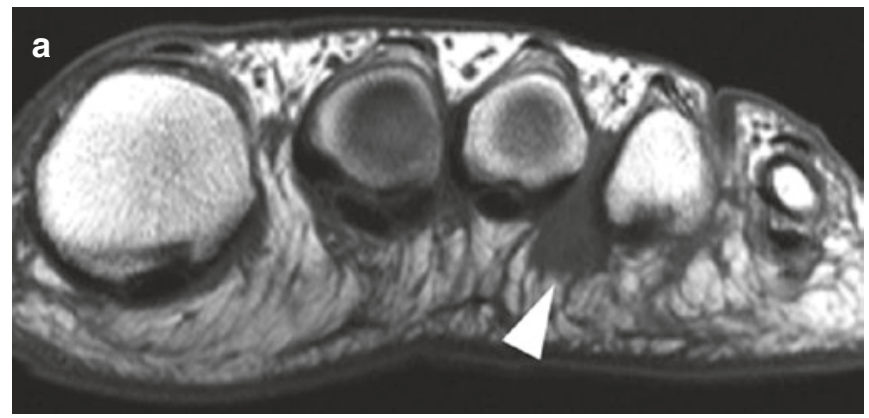

Fig. 8.14 Morton neuroma. Coronal T1- (a) and T2-weighted (b) MR images of the left forefoot obtained at the level of the metatarsal heads reveal a dumbbell-shaped soft tissue mass of low signal intensity pro- plantar aspect of the first and second toes. MR imaging can confirm the diagnosis by demonstrating denervation edema or fatty atrophy of the abductor hallucis, flexor digitorum brevis, flexor hallucis brevis, and first lumbrical muscles [32-34].

\subsubsection{Morton Neuroma}

Morton neuroma represents perineural fibrosis of a plantar digital nerve due to chronic entrapment. The lesion is most commonly found in the second or third intermetatarsal space at the level of the metatarsal heads deep to the transverse ligament. Clinical symptoms include numbness and forefoot pain radiating into the toes or leg. MR imaging shows a round or dumbbell-shaped mass extending from the involved intermetatarsal space in a plantar direction, typically exhibiting low T1- and T2-weighted signal intensity (Fig. 8.14). Lesion conspicuity can be increased by examination of the foot in prone position [33-35].

\section{Key Point}

- The diagnosis of nerve entrapment is based on clinical and imaging findings. MR images should be searched for morphologic changes of the involved nerve, signs of nerve compression, and muscle denervation.

\subsection{Concluding Remarks}

The complex anatomy and wide spectrum of pathology in the ankle and foot region present a significant challenge to the radiologist. Familiarity with the normal anatomy, anatomic variants, various clinical conditions, and their appearance on imaging is essential for accurate diagnosis.

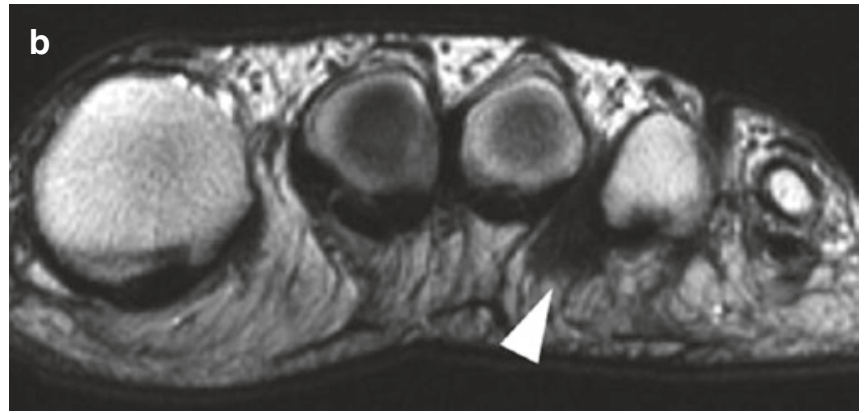

truding from the third intermetatarsal space into the plantar soft tissues (arrows) 


\section{Take Home Message}

- This short article can only give a rough overview on selected anatomic features and pathologies of the ankle and foot. Broaden your knowledge by studying major textbooks, book chapters, review articles, and scientific publications.

\section{References}

1. Albano D, Martinelli AD, Bianchi MN, et al. Posterior tibial tendon dysfunction: Clinical and magnetic resonance imaging findings having histology as reference standard. Eur J Radiol. 2018;99:55-61.

2. Flores DV, Gomez CM, Hernando MF, et al. Adult Acquired Flatfoot Deformity: Anatomy, Biomechanics, Staging. and Imaging Findings Radiographics. 2019;39:1437-60.

3. Ng JM, Rosenberg ZS, Bencardino JT, et al. US and MR imaging of the extensor compartment of the ankle. Radiographics. 2013;33:2047-64.

4. Schweitzer ME, Karasick D. MR imaging of disorders of the Achilles tendon. AJR Am J Roentgenol. 2000;175:613-25.

5. Taljanovic MS, Alcala JN, Gimber LH, et al. High-resolution US and MR imaging of peroneal tendon injuries--erratum. Radiographics. 2015;35:651.

6. De Maeseneer M, Marcelis S, Jager T, et al. Sonography of the normal ankle: a target approach using skeletal reference points. AJR Am J Roentgenol. 2009; 192:487-95.

7. Greaser MC. Foot and Ankle Stress Fractures in Athletes. Orthop Clin North Am. 2016;47:809-22.

8. Marshall RA, Mandell JC, Weaver MJ, et al. Imaging Features and Management of Stress, Atypical, and Pathologic Fractures. Radiographics. 2018;38:2173-92.

9. Spitz DJ, Newberg AH. Imaging of stress fractures in the athlete. Magn Reson Imaging Clin N Am. 2003;11:323-39.

10. Tafur M, Rosenberg RS, JBencardino JTMR. Imaging of the Midfoot Including Chopart and Lisfranc Joint Complexes. Magn Reson Imaging Clin N Am. 2017;25:95-12.

11. Linklater JM. Imaging of sports injuries in the foot. AJR Am J Roentgenol. 2012;199:500-8.

12. Castro M, Melao L, Canella C, et al. Lisfranc joint ligamentous complex: MRI with anatomic correlation in cadavers. AJR Am J Roentgenol. 2010;195:W447-55.

13. Raikin SM, Elias I, Dheer S, et al. Prediction of midfoot instability in the subtle Lisfranc injury. Comparison of magnetic resonance imaging with intraoperative findings. J Bone Joint Surg Am. 2009;91:892-9.

14. Hallinan J, Statum SM, Huang B, et al. High-Resolution MRI of the First Metatarsophalangeal Joint: Gross Anatomy and Injury Characterization. Radiographics. 2020;40:1107-24.

15. Yamada AF, Crema MD, Nery MD, et al. Second and Third Metatarsophalangeal Platar Plate Tears: Diagnostic Performance of Direct and Indirect MRI Features Using Surgical
Findings as the Reference Standard. AJR Am J Roentgenol. 2017;209:W100-8.

16. Hur ES, Bohl DD, Lee S. Lateral ligament instability: review of pathology and diagnosis. Curr Rev Musculoskelet Med. 2020;13:494-500.

17. Perrich KD, Goodwin DW, Hecht PJ, Cheung Y. Ankle ligaments on MRI: appearance of normal and injured ligaments. AJR. 2009;193:687-95.

18. Zoga AC, Schweitzer ME. Imaging sports injuries of the foot and ankle. Magn Reson Imaging Clin N Am. 2003;11:295-310.

19. Mengiardi B, Pinto C, Zanetti M. Medial collateral ligament complex of the ankle: MR imaging anatomy and findings in medial instability. Semin Musculoskelet Radiol. 2016;20:91-103.

20. Lee S, Lin J, Kamran JL, Bohl DD. Deltoid ligament rupture in ankle fracture: diagnosis and management. J Am Acad Orthop Surg. 2019;27:e648-58.

21. Linklater J. Ligamentous, chondral and osteochondral ankle injuries in athletes. Semin Musculoskelet Radiol. 2004;8:81-98.

22. Sharif B, Welck M, Saifuddin A. MRI of the distal tibiofibular joint. Skelet Radiol. 2020;49:1-17.

23. Oae K, Takao M, Maito K, et al. Injury of the tibiofibular syndesmosis: value of MR imaging for diagnosis. Radiology. 2003;227:155-61.

24. O'Loughlin PF, Heyworth BE, Kennedy JG. Current concepts in the diagnosis and treatment of osteochondral lesions of the ankle. Am J Sports Med. 2010;38:392-404.

25. Kirschke J, Braun S, Baum T, et al. Diagnostic value of CT arthrography for evaluation of osteochondral lesions at the ankle. Biomed Res Int. 2016;3594253:2016.

26. Berndt AL, Harty M. Transchondral fractures (osteochondritis dissecans) of the talus. JBJS Am. 1959;41:988-1020.

27. Loomer R, Fisher C, Lloyd-Smith R, Sisler J, Cooney $\mathrm{T}$. Osteochondral lesions of the talus. Am J Sports Med. 1993;21:13-9.

28. Sellon E, Robinson P. MR imaging of impingement and entrapment syndromes of the foot and ankle. Magn Reson Imaging Clin N Am. 2017;25:145-58.

29. Huh YM, Suh JS, Lee JW, Song HT. Synovitis and soft tissue impingement of the ankle: assessment with enhanced threedimensional FSPGR MR imaging. J Magn Reson Imaging. 2004;19:108-16.

30. Cerezal L, Abascal F, Canga A, et al. MR imaging of ankle impingement syndromes. Am J Roentgenol AJR. 2003;181:551-9.

31. Robinson P, White LM, Salonen DC, Daniels TR, Ogilvie-Harris D. Anterolateral ankle impingement: MR arthrographic assessment of the anterolateral recess. Radiology. 2001;221:186-90.

32. Default EM, Demondion X, Bieganski A, Thiron MC, Mestdagh H, Cotton A. Imaging of foot and ankle nerve entrapment syndromes: from well-demonstrated to unfamiliar sites. Radiographics. 2003;23:613-23.

33. Ferkel E, Davis WH, Ellington JK. Entrapment neuropathies of the foot and ankle. Cin Sports Med. 2015;34:791-801.

34. Beltran LS, Bencardino J, Ghazikhanian V, Beltran J. Entrapment neuropathies III: lower limb. Semin Musculoskelet Radiol. 2010;14:501-11.

35. Woertler K. Tumors and tumor-like lesions of peripheral nerves. Semin Musculoskelet Radiol. 2010;14:547-58.

Open Access This chapter is licensed under the terms of the Creative Commons Attribution 4.0 International License (http://creativecommons. org/licenses/by/4.0/), which permits use, sharing, adaptation, distribution and reproduction in any medium or format, as long as you give appropriate credit to the original author(s) and the source, provide a link to the Creative Commons license and indicate if changes were made.

The images or other third party material in this chapter are included in the chapter's Creative Commons license, unless indicated otherwise in a credit line to the material. If material is not included in the chapter's Creative Commons license and your intended use is not permitted by statutory regulation or exceeds the permitted use, you will need to obtain permission directly from the copyright holder. 